\title{
Post Traumatic Scar with Repeated Trophic Ulcers Treated With a Cocktail of Ablative Pixel Erbium YAG Laser, PRP Therapy and Autologous Fat Transplant - A Novel Case Report of Terminal Nerve Ending Regeneration through Regenerative Surgery
}

Garg S*

Department of Dermatology, Cosmetic Surgery and Intervention Dermatology, Aura Skin Institute, Chandigarh, India

\begin{abstract}
This report discusses the case of a twenty-eight years old male patient reported in our tertiary care skin institute for the treatment of post-traumatic scar revision on his left heel following a history of road side accident one year back. The treatment of this patient is based on the principle of regenerative surgery where body's own tissues and fluids are utilized for healing and repair. The intention of combining three procedures was to induce repeated injuries on scarred skin and give appropriate direction for wound repair in presence of PRP released growth factors. Eventually by the end of third session, the patients started perceiving touch and pain sensation which led to complete healing of trophic ulcers and prevented risk of osteomyelitis to underlying bone. Author strongly believes that no amount of external agents can ever match our body's own healing potential; all it needs is a little support, right nutrition and the direction to work in.
\end{abstract}

Keywords: Trophic ulcer; Pixel ErYAG laser; Platelet rich plasma therapy; Autologous fat transplant; Regenerative surgery

\section{Introduction}

A twenty-eight years old male patient reported in our tertiary care skin institute for the treatment of post-traumatic scar revision on his left heel following a history of road side accident one year back. The area healed as hyper pigmented scar along with complete loss of sensation secondary to crushing injury to the cutaneous sural and tibial nerves $(\mathrm{S} 1, \mathrm{~S} 2)$ thus leading to repeated trophic ulcers over sole. The treatment of this patient was planned on the principle of regenerative surgery where body's own tissues and fluids are utilized for healing and repair process. The intention of combining three procedures was to induce repeated injuries on scarred skin and give appropriate direction for wound repair in presence of PRP released growth factors; autologous fat acted as a reservoir of adipocytes besides proving cushion over bony areas. Adiposite-derived stem cells (ASC) help in differentiation, migration and proliferation of endothelial cells in the presence of various cytokines and proinflammatory mediators from PRP therapy.

\section{Case Presentation}

A twenty-eight years old male patient reported in our tertiary care skin institute for the treatment of post-traumatic scar revision on his left heel following a history of road side accident one year back. At the time of accident, the heel area of sole got crushed completely and he was given full thickness pedicle graft from thigh muscle by the operating surgeon. The area healed as hyper pigmented scar along with complete loss of sensation secondary to crushing injury to the cutaneous sural and tibial nerves (S1, S2) thus leading to repeated trophic ulcers over sole.

\section{Treatment plan}

We planned to resurface the scar with pixel ErYAG Laser at Harmony XL platform from Alma Lasers and combined injectable PRP therapy in every session for 5 sequential sessions, placed at a gap of one month each. The intension was to ablate the scarred skin and make new skin in presence of active growth factors from PRP therapy which aid in wound repair. The laser was done at a fluence of $1400 \mathrm{mj} / \mathrm{p}$ with 5 passes each at long pulse mode. PRP was prepared by taking $13.5 \mathrm{cc}$ of venous blood from patient's antecubital vein, mixed with $1.5 \mathrm{cc}$ of ACD-A solution as anticoagulant in YCell Bio kit and centrifuged in Remi centrifuge at $3200 \mathrm{rpm}$ for 4 minutes by single spin method. We collected $3 \mathrm{cc}$ of platelet rich plasma along with buffy coat ready to be injected; since there were multiple pricks, hence no activator was used. The blood oozing from pricks triggered the degranulation of platelets leading to release of millions of growth factors. With each subsequent session there were signs of improvement in texture of scar and the frequency of trophic ulcers went down as shown in Figure 1. A tiny $2.5 \mathrm{~mm}$ skin biopsy tissue was sent for histopathological examination before the start of first session and findings are shown in Figure 2. By the end of third session patient started reporting appreciation of touch and pain sensations which was completely absent at the time of start of surgery and was the principal cause of getting repeated trophic ulcers. The patient was given $15 \mathrm{cc}$ of autologous fat transplant in sub cutis, harvested from medial side of thigh under $2 \%$ injectable lignocain solutions in fourth session along with other two procedures. This helped in cushioning the skin directly overlying the calcaneus bone, thus minimizing the compression trauma. The awareness of pain sensation on overexertion helped him curb his movement, hence reducing the incidence of repeated trophic ulcers. By the end of 5 sessions, there was no trophic ulcer seen and the patient was extremely satisfied with the outcome and overall there was $40 \%$ improvement in touch and pain sensation on clinical examination.

*Corresponding author: Dr. Suruchi Garg, Department of Dermatology, Cosmetic Surgery and Intervention Dermatology, Aura Skin Institute, Chandigarh, India, Tel: 0172274 8888; E-mail: gargsuruchi01@gmail.com

Received October 28, 2017; Accepted November 13, 2017; Published November 16, 2017

Citation: Garg S (2017) Post Traumatic Scar with Repeated Trophic Ulcers Treated With a Cocktail of Ablative Pixel Erbium YAG Laser, PRP Therapy and Autologous Fat Transplant - A Novel Case Report of Terminal Nerve Ending Regeneration through Regenerative Surgery. J Mol Genet Med 11: 303 doi:10.4172/1747. 0862.1000303

Copyright: (c) 2017 Garg S. This is an open-access article distributed under the terms of the Creative Commons Attribution License, which permits unrestricted use, distribution, and reproduction in any medium, provided the original author and source are credited 
Citation: Garg S (2017) Post Traumatic Scar with Repeated Trophic Ulcers Treated With a Cocktail of Ablative Pixel Erbium YAG Laser, PRP Therapy and Autologous Fat Transplant - A Novel Case Report of Terminal Nerve Ending Regeneration through Regenerative Surgery. J Mol Genet Med 11: 303 doi: 10.4172/1747-0862.1000303

Page 2 of 2

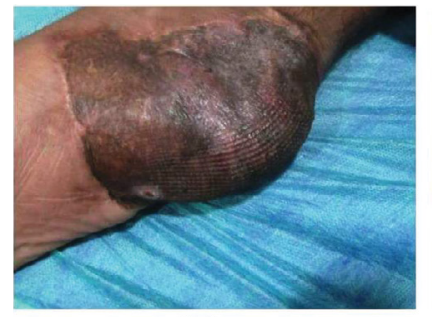

Before

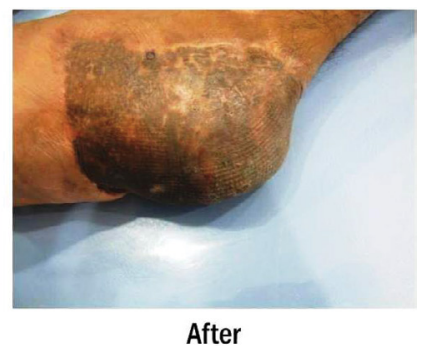

After
Figure 1: Post-traumatic hyper pigmented scar over heel with recurrent trophic ulcer treated with a cocktail of pixel ErYAG laser, PRP therapy and autologous fat grafting. The second picture shows less pigmented and healthier skin with healed trophic ulcer and improved touch and pain sensation.

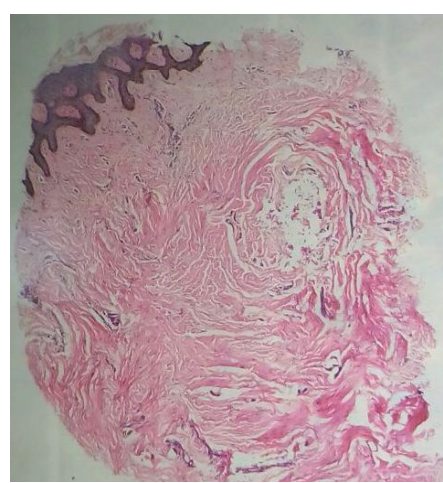

Figure 2: Histopathological section shows keratinized stratified squamous epithelium with over activity of mealnocytes at basal cell layer. Dermis shows thick fibro-collageneous tissue with mild inflammatory infiltrate and hemorrhages (H\&E Stain, 20x).

\section{Discussion}

The treatment of this patient is based on the principle of regenerative surgery where body's own tissues and fluids are utilized for healing and repair. The intention of combining three procedures was to induce repeated injuries on scarred skin and give appropriate direction for wound repair in presence of PRP released growth factors; autologous fat acted as a reservoir of adipocyte-derived stem cells (ASC) besides proving cushion over bony areas. Adiposite-derived stem cells (ASC) help in differentiation, migration and proliferation of endothelial cells in the presence of various cytokines and pro-inflammatory mediators from PRP therapy like platelet derived growth factor (PDFG), vascular endothelial growth factor (VEGF), transforming growth factor $-b$ (TGF-b), fibroblast growth factor (FGF), insulin growth factor (IGF), stem cell factor (SCF), nerve growth factor (NGF) etc. [1,2]. Already there is supportive literature highlighting the role of PRP therapy in improving microcirculation, collagen remodeling thus improving skin texture and supporting appendage growth specially that of hair follicles $[3,4]$. Since the PRP therapy has been proven to improve viability of hair follicles, it can act on other structures of skin and other organs as well to renew and regenerate their structural and functional capacity. The interplay of cascade of positive signals in presence of ASC and PRP therapy helps in improving overall skin milieu, sub cutis and extracellular matrix environment for better cell and tissue survival $[2,5]$.

Cervelli et al. have used a combination of non-ablative erbium glass laser, PRP therapy admixed with fat grafting for post traumatic scars and found the combination of three superior to the use of monotherapy with laser alone or with a combination of PRP and fat grafting [6]. The ablative lasers give the coagulation and degradation effect to extrude the scarred tissue besides photodynamic, photochemical, photo enzymatic stimulation leading to repair and regeneration [7]. In our case report, we have observed that the ablation of scarred skin with pixel ErYAG laser and supporting the re-epithelisation in presence of cytokines, growth factors, molecular signals and cells helped in creating near normal skin on one hand and autologous fat transplant helped in providing a cushion over bony prominences besides providing vital adipositederived stem cells reservoir that aided in improving microcirculation and scar remodeling. Since patient reported nearly $40 \%$ improvement in appreciation of touch and pain sensation on clinical examination, which was completely absent prior to start of treatment, it also helped him preventing repeated injuries thus taking care of the underlying etiological factor for trophic ulcer.

It will not be an overstatement that platelet rich plasma contains a growth factor for practically each and every cell in skin and sub cutis. PRP with autologous fat graft have been documented to improve dental pain, due to the regenerative role of the stromal fraction of adipose tissue and adipose-derived stem cells leading to axon regeneration thus reducing nerve excitatory pattern with consequent pain control [8]. The nerve growth factor (NGF) and neuroimmunophilin ligand in presence of other supportive cytokines helped in repairing the damaged nerve twigs and endings of post tibial and sural nerves in skin tissue, secondary to repeated stimulus to heal from injury induced by ablative laser in our case. Eventually by the end of third session, the patients started perceiving touch and pain sensation which led to complete healing of trophic ulcers and prevented risk of osteomyelitis to underlying bone. To the best of our knowledge the treatment of post traumatic trophic ulcers has not been reported with combination of these three techniques taking regenerative surgery as the principle of healing.

\section{Conclusion}

Author strongly believes that no amount of external agents can ever match our body's own healing potential; all it needs is a little support, right nutrition and the direction to work in. Though more elaborate randomized controlled trials are needed to substantiate but our case report gives a new concept of renewing and regenerating scarred skin with damages nerves by surgically correct ablative lasers in presence of body's own supportive pro-inflammatory cytokines from PRP and cushioning with adiposite derived stem cell network.

\section{References}

1. Reinders Y, Felthaus O, Brockhoff G, Pohl F, Ahrens N, et al. (2017) Impact of platelet-rich plasma on viability and proliferation in wound healing processes after external radiation. Int J Mol Sci 18: 1819

2. Haubne F, Muschte D, Schuster N, Pohl F, Ahrens N, et al. (2015) Platelet-rich plasma stimulates dermal microvascular endothelial cells and adipose derived stem cells after external radiation. Clin. Hemorheol. Microcirc 61: 279-290.

3. Garg S, Manchanda S (2017) Platelet-rich plasma-an 'Elixir' for treatment of alopecia: personal experience on 117 patients with review of literature. Stem Cell Investig 18: 64.

4. Garg S (2016) Outcome of intra-operative injected platelet-rich plasma therapy during follicular unit extraction hair transplant: A prospective randomised study in forty patients. J Cutan Aesthet Surg 9: 157-164.

5. Smith OJ, Kanapathy M, Khajuria A, Prokopenko M, Hachach-Haram N, et al. (2017) Protocol for a systematic review of the efficacy of fat grafting and platelet-rich plasma for wound healing. Syst Rev 6: 111.

6. Cervelli V, Nicoli F, Spallone D, Verardi S, Sorge R, et al. (2012) Treatment of traumatic scars using fat grafts mixed with platelet-rich plasma, and resurfacing of skin with the $1540 \mathrm{~nm}$ non-ablative laser. Clin Exp Dermatol 37: 55-61.

7. Nita AC, Orzan OA, Filipescu M, Jianu D (2013) Fat graft, laser $\mathrm{CO}_{2}$ and platelet-rich plasma synergy in scars treatment. J Med Life 6: 430-433

8. Khourey JI, Avaida C, Nasr M, Hokayem N (2017) Platelet-rich plasma and fat grafting for the treatment of inferior alveolar nerve neuropathy: The first case report. Oral Maxillofacial Surg Cases 3: 107-111. 